\title{
„Sie Lümmel, Sie!“ Kleine Typologie sozialer Kategorisierungen „frecher" Jugendlicher
}

\section{Zur Einführung}

Im Kontext eines DFG-geförderten Projekts beschäftigen wir $^{1}$ uns mit dem Umgang Jugendlicher mit sprachlicher Höflichkeit heute (dazu Neuland 2013, 2017). Das Thema hat eine lange Tradition in der Sprach- und Kulturgeschichte: Immer wieder hat das Verhalten Jugendlicher, besonders gegenüber Erwachsenen, Kritik an ihrer (vermeintlichen) Unhöflichkeit ausgelöst und wurde als Zeichen eines Sprach- und Sittenverfalls gebrandmarkt. Eine ganze Liste von Ausdrücken lässt sich zusammentragen, die im Laufe der Sprachgeschichte als soziale Kategorisierungen aus einer Außensicht für freche, ungezogene Jugendliche geprägt wurden:

Bengel, Bursche, Bube, Flegel, Frechdachs, Früchtchen, Halbstarker, Knilch, Lausbub, Lausebengel, Lümmel, Rabauke, Raufbold, Rotzlöffel, Rowdy, Rüpel, Schlingel, Strolch ...

Die Liste, die sich interessanterweise nur auf männliche Jugendliche bezieht, lässt sich fortsetzen. Korrespondierende Bezeichnungen für Mädchen sind hingegen kaum zu finden. ${ }^{2}$ Solche geschlechtstypischen Rollenklischees sind mit Konnotationen verbunden wie:

flegelhaft, frech, grob, roh, rüde, rüpelhaft, vorlaut, unartig, unerzogen, ungehobelt, ungezogen, unhöflich.

Einige dieser Ausdrücke erscheinen antiquiert und sind heute ungebräuchlich geworden. Wann hört man heute noch die Zurechtweisung: „Sie Lümmel, Sie!“,

1 DFG-Projekt Sprachliche Höflichkeit bei Jugendlichen. Empirische Untersuchungen zu Gebrauchs- und Verständnisweisen im Schulalter, Projektleitung: Eva Neuland, wissenschaftliche Mitarbeiter: Benjamin Könning und Elisa Wessels (www.hoeflichkeit.uni-wuppertal.de, Stand: 12.1.2018).

2 Allein Spreckels widmet sich in ihrer Studie von 2006 Klassifikationsausdrücken für Mädchen aus der Sicht einer heutigen Mädchengruppe. 
und welcher historische und situative Kontext wird dabei konstruiert? Dieser kleine Beitrag will mit lexikographischen und korpusanalytischen sowie pragmatischen Ausflügen einige sprach- und kulturhistorische Anmerkungen zu diesen Fragen vorstellen und wird sich dabei auf die folgenden vier ausgewählten Beispiele beschränken: Bengel, Flegel, Lümmel und Rüpel.

\section{Rückblick in die lexikographische Sprachgeschichte}

\subsection{Historische Belege}

Betrachten wir zunächst einige historische Belege und beginnen mit dem Deutschen Wörterbuch von Grimm (1854ff.). Hier wird nach der Ausgabe von 1971 aufgeführt:

\section{Bengel:}

Neben der Erstbedeutung: knüttel zum schlagen, werfen wird als Zweitbedeutung aufgeführt: schimpfwort mit der bedeutung von homo agrestis, rusticus, zumal ein junger, langer aufschüszling, und oft gutmütig genommen.

Unter den Beispielen findet sich Schuppius (Streitschrift 1,82, 1659): der bengel, der noch in die schul gegangen und mit ruthen gestrichen worden ist, als ich in einem vornehmen ehrenstand gesessen habe, gehet mit mir umb, als ob ich mit ihme die schweine gehütet hätte.

\section{Flegel:}

Neben der Erstbedeutung: Gerät zum Getreide Schlagen, Dreschen bezieht sich die Zweitbedeutung auf die Übertragung auf menschliche Eigenschaften: schelte für bauer, rusticus, agrestis.

Dazu ein Zitat von Weise (Esau und Jacob, 1696): ich meinte ein solcher flegel sollte sich besser unter das commando schicken.

Als Drittbedeutung soll: penis nicht unterschlagen werden.

\section{Lümmel:}

Lümmel wird bei Grimm als flegelhafter mensch erläutert, was seit Mitte des 17. Jahrhunderts als oft gebrauchtes, in allen Wörterbüchern aufgeführtes Wort erscheine. Und ein Zitat aus Wieland (Pervonte oder die Wünsche, 1855) lautet: dasz sie (die mutter), mit aller ihrer müh, aus ihrem lieben sohn Pervonte nichts ziehen, und zu nichts den lümmel brauchen konnte.

Auch wird erwähnt, dass das Wort in einigen Redensarten des 17. Jahrhunderts im Sinne von buhlerei, bemühung um weibliche Zuneigung verwendet wurde.

\section{Rüpel:}

Nach dem Grimmschen Wörterbuch Koseform des Bauernknechts Ruprecht, ist die älteste bezeugte Bedeutung: ungeschliffener, grober mensch, teils mit den Attributen: lustig, lie- 
derlich, unstät, Rüpel für einen mutwilligen, unruhigen buben, mit Bezug auf das Aussehen auch ein schwarzer, unreinlicher mensch.

Werfen wir noch einen Blick in die Etymologie, wie sie in Kluges Etymologischem Wörterbuch der deutschen Sprache (bearbeitet von Seebold) 2002 verzeichnet ist:

\section{Bengel:}

Bengel wird in der Bedeutung: ungezogener Junge aufgeführt. Nach dem Verweis auf die ursprüngliche Bedeutung (13. Jh.) heißt es: Die Übertragung auf Menschen stellt diese (ähnlich wie bei Flegel) als Menschen, die mit einem groben Bengel hantieren, und deshalb als „grob“ dar. Auch hier folgt der Hinweis auf männliches Glied. (ebd.: 97)

\section{Flegel:}

Nach der Erstbedeutung (10. Jh.) als Schimpfwort zunächst gebraucht für den Bauern nach seinem typischen Arbeitsgerät. Angeführt wird auch das Verb sich (hin)flegeln. (ebd.: 271)

\section{Lümmel:}

Das Wörterbuch verweist auf die Herkunft zum mhd. lüemen erschlaffen (16. Jh.) und geht nicht auf die soziale Kategorisierung ein.

Auch hier wird ein Verb, und zwar (herum)lümmeln aufgeführt. (ebd.: 527)

\section{Rüpel:}

Auch bei diesem Ausdruck bleibt das Wörterbuch bei der ursprünglichen Etymologie (16. Jh). (ebd.: 697)

Halten wir als kurzes Zwischenfazit fest, dass die den sozialen Kategorien zugeordneten Attribute wie: grob, roh, rüde, ungehobelt, ungeschliffen im Fall von Bengel und Flegel aus der ursprünglichen Bedeutung als bäuerliche Arbeitsgeräte abgeleitet werden können. Auch die weitere Bedeutungsvariante Penis hängt damit zusammen. Die Etymologie von Rüpel erklärt sich durch die einem Bauernknecht zugeschriebenen Merkmale, die sich in der heutigen sozialen Kategorie noch wiederfinden lassen. Allein beim Lümmel kann bei der Familienherkunft aus erschlaffen, schlaff, matt auf eine gewisse semantische Ähnlichkeit zu: Formlosigkeit, Sich Gehenlassen, im weiteren und im übertragenen Sinn vielleicht auch Unerzogenheit geschlossen werden. Jedenfalls erscheinen alle vier Bezeichnungen sprachgeschichtlich bereits als Pejorativa (vgl. dazu Finkbeiner/Meibauer/Wiese (Hgg.) 2016).

Da sich alle vier ausgewählten Ausdrücke sprachgeschichtlich auf männliche Jugendliche beziehen, deren Verhalten als nicht der üblichen Gepflogenheit entsprechend aufgefasst wurde, liegt es nahe, nach ihrem Vorkommen in Wörterbüchern für bestimmte jugendliche Zielgruppen zu fragen. Eine Recherche in Wörterbüchern der historischen deutschen Studentensprache führt jedoch $\mathrm{zu}$ folgendem aufschlussreichen Ergebnis:

In Kluges „Deutscher Studentensprache“ (1895/1984) wird nur der Ausdruck: Rüpel in der Bedeutung: Schlingel, Lümmel aufgeführt. Und im: Burschicosen 
Wörterbuch eines Verfassers mit dem Pseudonym Vollmann (1846) findet sich nur der Flegel mit den Bedeutungen: ein roher Kerl, Holzkopf, Grobian Nro 1, Esel, Stier. Schuchardts „Studentikoses Conversationslexikon“ (1825/1984) verzeichnet keinen der Ausdrücke.

Dies aber kann nur bedeuten, dass diese Ausdrücke durchaus nicht als typisch studentensprachlich angesehen wurden und keine Selbstbezeichnungen bzw. Ethnokategorien darstellen, sondern Fremdbezeichnungen, wie sie eventuell auch eher für nicht-akademische, bäuerliche Jugendliche verwendet wurden. Darüber fehlen aber leider lexikographische Aufzeichnungen.

\subsection{Aktuelle Bedeutungen}

Bevor wir uns in zeitgenössischen Lexika vergewissern, lohnt ein Blick in schülersprachliche Wortsammlungen, wie sie von Küpper in den 1970er Jahren vorgelegt wurden. In Küppers „Jugenddeutsch“, dem Band 6 des Wörterbuchs der deutschen Umgangssprache (1970), ist allerdings keiner der vier Ausdrücke aufgeführt. Das unterstreicht die obige Vermutung nun auch in schülersprachlicher Hinsicht.

In Küppers später erschienenem „Illustrierten Lexikon der deutschen Umgangssprache“ (1982) finden wir in verkürzter Form:

Bengel: ungezogener Junge; eingetragen ist auch das Verb: bengeln: verprügeln. (ebd.: 342)

Lümmel: flegelhafter Halbwüchsiger; mutwilliger, frecher junger Mann; Lümmel von der ersten Bank, im Zusammenhang mit den „Lümmel-Pauker-Filmen“ nach 1966. Eingetragen ist auch das Verb: lümmeln: müßiggehen, sich lümmeln: sich ungesittet benehmen, sich flegeln. (ebd.: 1811)

Rüpel: grober, ungesitteter Mann. Eingetragen ist u.a. auch das Verb: rüpeln i.S.v. sich grob benehmen oder äußern. (ebd.: 2364)

Die Figur der Lümmel erscheint in den 1960er Jahren im Kontext der Schule als Konterpart der Paukerfigur. Nach Vorlagen von Romanen von Heinrich und Alexander Spoerl und Erich Kästner entstanden in den sechziger und siebziger Jahren des letzten Jahrhunderts eine Reihe von „Penner“- und „Paukerfilmen“ mit den Motiven des Verulkens und Verspottens der Lehrerfiguren, wie schon bei Max und Moritz. Eine Bubengeschichte in sieben Streichen (erstmals 1865) von Wilhelm Busch vorgeprägt. Die „Lausbuben“ Max und Moritz haben eine Fortsetzung in den „Lümmeln von der ersten Bank“ (1968) gefunden. 


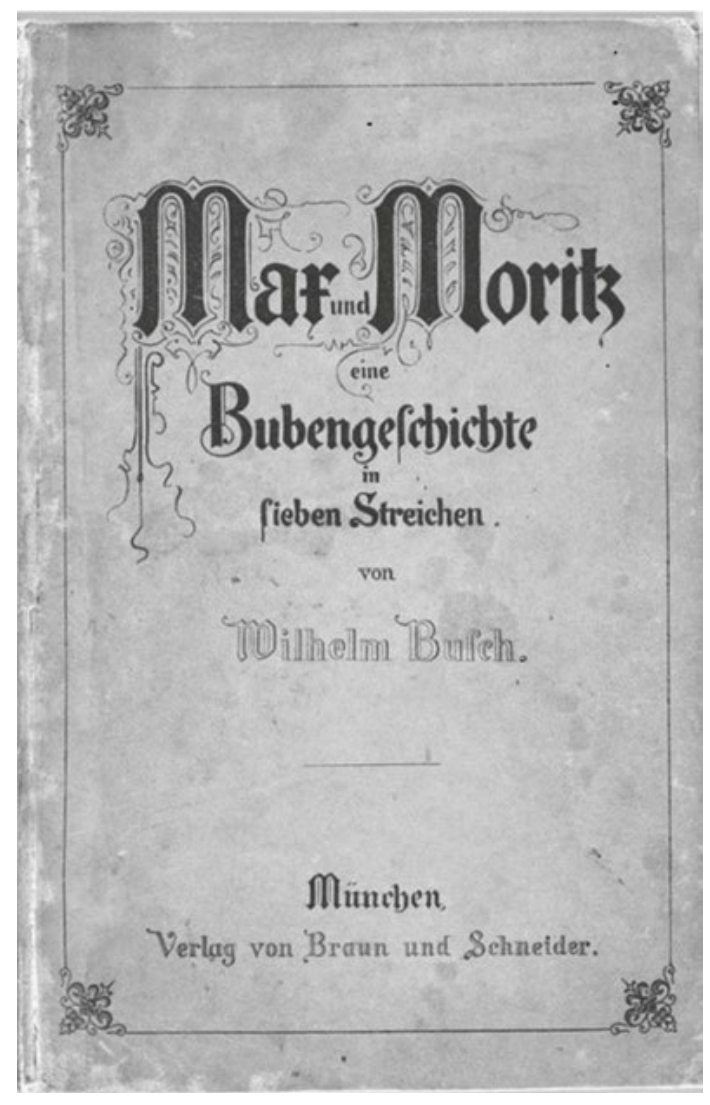

Abb. 1: Wilhelm Busch - Max und Moritz (1865)

Wie die folgenden aktuellen Eintragungen belegen, sind die Ausdrücke im Wesentlichen in dieser Bedeutung in die zeitgenössischen Wörterbücher der deutschen Sprache eingegangen, so in die Neuausgabe von Wahrig-Burfeind (Hg.) (2014):

Bengel: (umg.) (frecher) Junge; aber auch: kleiner, hübscher, süsser Bengel; er ist ein fauler, ungezogener Bengel

Flegel: (abwertend) grober, unerzogener Mensch, Lümmel; er ist ein unverschämter, richtiger Flegel

Lümmel: (umg.) ungezogener, grober, frecher Mensch, Flegel

Rüpel: Mensch, der sich rüpelhaft benimmt, Flegel, Grobian; so ein Flegel! 
Im Duden-Online-Wörterbuch (Stand: Februar 2017) findet man schließlich:

Bengel: (frecher) junger Bursche, Halbwüchsiger; (familiär) niedlicher kleiner Junge

Flegel: (abwertend) (junger) Mann, der als ungeschliffen, schlecht erzogen angesehen wird; Lümmel

Lümmel: (abwertend) (junger) Mann, der als frech, ungezogen, als Person mit flegelhaftem Benehmen angesehen wird; (umg., fam.) Bursche, Kerl

Rüpel: (abwertend) männliche Person, die ungehobelt und grob ist, die sich schlecht, ungezogen benimmt, deren Betragen andere empört.

Halten wir als Fazit fest, dass die vier Ausdrücke weitgehend bedeutungsgleich als pejorative Bezeichnungen mit den dominierenden semantischen Merkmalen frech und ungezogen beschrieben werden. Bei Bengel ist eine personale Referenz auf kleiner Junge sowie eine positive familiäre Nebenbedeutung verzeichnet.

Die lexikographischen Daten sagen allerdings noch nichts über den tatsächlichen Gebrauch solcher Ausdrücke aus. Die wenigen angeführten Beispiele wie: er ist ein richtiger Flegel, so ein Flegel! betreffen konstatierende und exklamatorische Sprechakte, in denen die sozial abwertende Attribuierung einer nicht anwesenden Person ausgedrückt wird. Aktuelle gebrauchsbasierte Daten aus der gesprochenen Alltagssprache liegen zu diesen Ausdrücken aber nicht vor. Einschlägige Forschungsbeiträge aus der linguistischen Pragmatik und der Soziolinguistik betten das von Sacks und Schegloff entwickelte Konzept: membership categorization (vgl. Schegloff 2007) in den Interaktionsprozess ein.

Deshalb wollen wir uns in einem zweiten Schritt korpusanalytischen Beobachtungen zuwenden, um mehr über schriftliche Verwendungskontexte der vier Klassifikationsausdrücke in Erfahrung bringen zu können.

\section{Korpusanalytische Beobachtungen}

Dazu greifen wir mit Hilfe des korpuslinguistischen Instrumentariums COSMAS II auf das DEREKo, das Deutsche Referenzkorpus der geschriebenen Gegenwartssprache des Instituts für Deutsche Sprache zurück. Es umfasst mit über 31 Milliarden Wörtern aus Texten der Belletristik, Wissenschaft und Öffentlichkeit, v.a. Zeitungstexte der Gegenwart und jüngsten Vergangenheit. 


\subsection{Frequenz und Produktivität}

Fragen wir zunächst nach Frequenz und Produktivität der vier sozialen Kategorien, so erhalten wir folgenden Befund:

Tab. 1: Frequenz und Produktivität

\begin{tabular}{llll}
\hline Ausdruck & $\begin{array}{l}\text { Simplizia } \\
\text { (tokens) }\end{array}$ & $\begin{array}{l}\text { Anzahl der } \\
\text { Wortformen (types) }\end{array}$ & $\begin{array}{l}\text { Vorkommenshäufigkeit des Wortes } \\
\text { in allen Wortformen (tokens) }\end{array}$ \\
\hline Bengel* $^{*}$ & 5509 & $1062^{\star \star}$ & 20.734 \\
\hline Flegel* $^{*}$ & 3941 & $1025^{\star \star}$ & 24.061 \\
\hline Lümmel & 1451 & 669 & 9594 \\
\hline Rüpel & 2412 & $1463^{\star \star \star}$ & 45.819 \\
\hline
\end{tabular}

* tritt auch als Eigenname auf

** Morphemgrenzen sind nicht berücksichtigt worden

$\star \star \star$ Umlaute sind nicht berücksichtigt worden

Hier zeigt sich, dass der Ausdruck Flegel besonders häufig in allen Wortformen und auch besonders produktiv erscheint. Diese Zahlen sind allerdings noch zu bereinigen, und zwar treten einerseits einige der Ausdrücke auch als Eigenname auf, und andererseits bleiben Morphemgrenzen unberücksichtigt (bei Flegel z.B. pflegeleicht). Ähnliches gilt für Bengel und Rüpel, die auch als Eigenname und mit unberücksichtigten Morphemgrenzen sowie Umlauten auftreten können (z.B.: Silbengelenk, Skrupel).

Trotz dieser Ungenauigkeiten mögen den folgenden Verlaufskurven dennoch einige aufschlussreiche Tendenzen im Hinblick auf die zeitlichen Verläufe entnommen werden: So ist trotz der o.g. Einwände erkennbar, dass der Ausdruck Lümmel (siehe Abb. 4) die höchste Auftretenshäufigkeit in den 1960er Jahren verzeichnet, die anschließend kontinuierlich abnimmt. Die Gebrauchsfrequenzen der Ausdrücke Bengel, Flegel und Rüpel hingegen weisen ab der Jahrtausendwende einen Anstieg auf, der Rüpel (siehe Abb. 5) ist bis in die heutige Zeit zunehmend aktuell. 


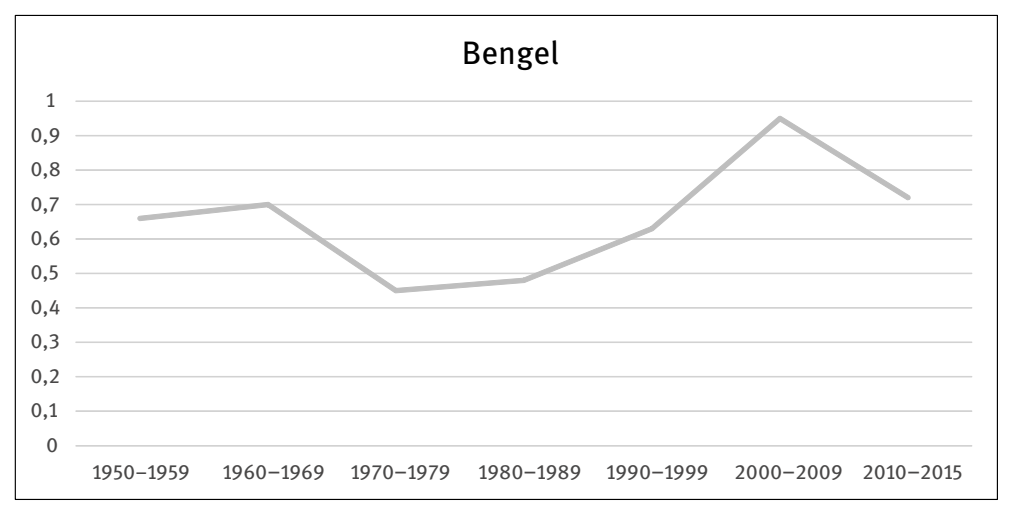

Abb. 2: Bengel (Simplizium, diachron)

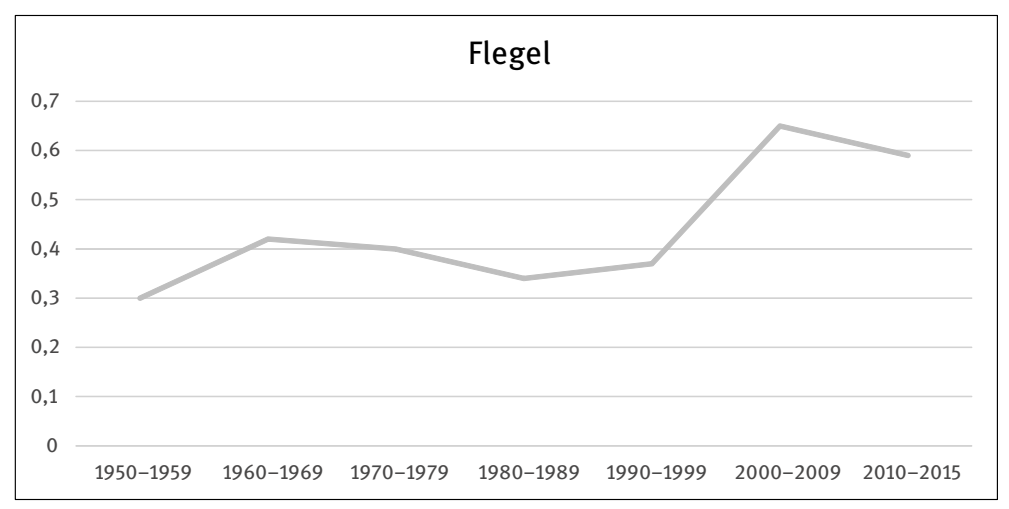

Abbildung 3: Flegel

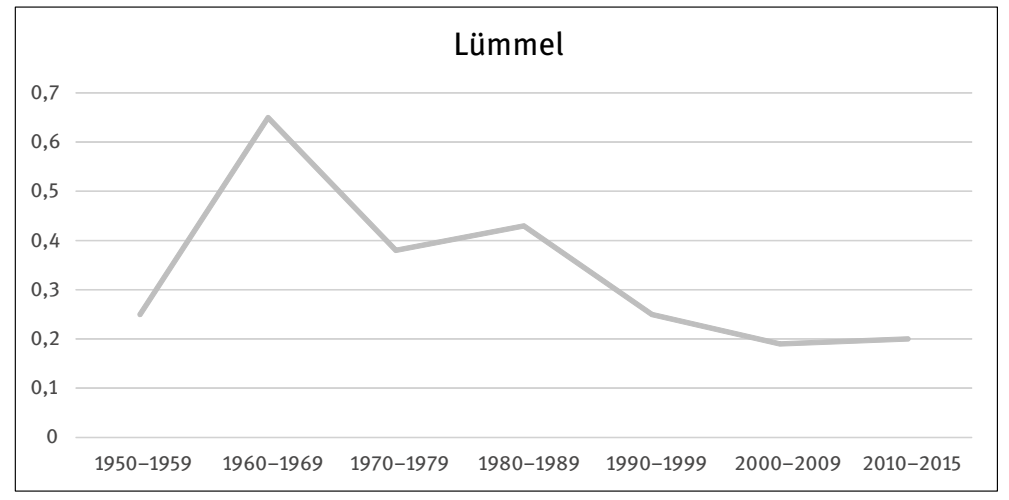

Abb. 4: Lümmel 


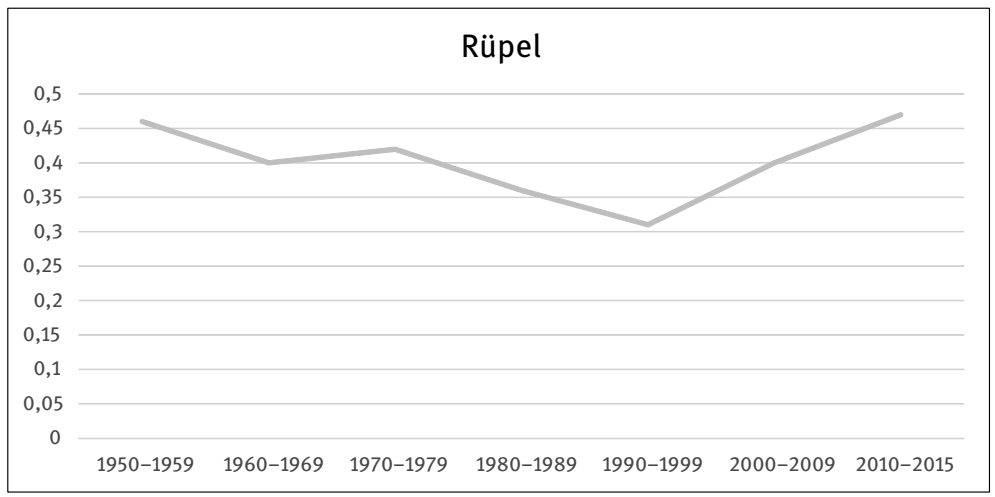

Abb. 5: Rüpel

\subsection{Kontexte und Kookkurrenzen}

Für die Identifikation der Kookkurrenzen wurde eine Spannweite von fünf Wörtern und maximal einem Satz ausgewählt. Die Ergebnisse werden in Form von Wordle-Darstellungen ${ }^{3}$ präsentiert:

\subsubsection{Bengel}

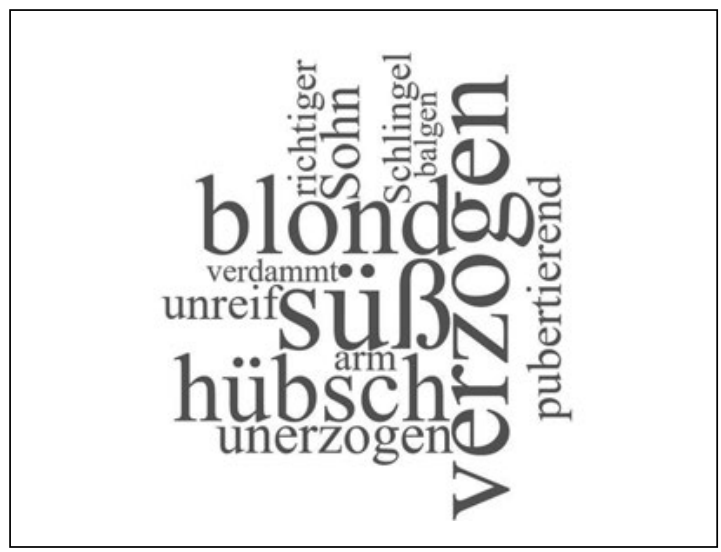

Abb. 6: Bengel

3 Methode zur Visualisierung von Vorkommenshäufigkeiten und Kookkurenzen eines Lexems. 
So zeigt sich bei Bengel mit den stark vertretenen positiven Attributen: blond, süß, hübsch sowie den negativen Wertungen: verzogen, unerzogen, unreif, pubertierend die Altersdimension junges Kind und die positive, auch mit dem Ausdruck Schlingel verbundene Bedeutungsdimension, wie sie schon in den Bedeutungszuschreibungen der Wörterbücher zu erkennen waren.

Diese Gebrauchskontexte werden auch durch die beiden folgenden Textbeispiele aus der Tagespresse zu verschiedenen Epochen veranschaulicht:

Beispiel 1: Bengel:

\section{Die Zeit, 5.12.1957: 38; Kinder im Stadtrat}

Kindern und Jugendlichen kann man nicht leicht etwas vormachen. Sie sind kritische Beobachter und unbestechliche Richter. Die Erwachsenen könnten manchmal von ihnen lernen. Aber sie sagen „Geht mal raus, Kinder!“, wenn sie zweifelhafte Dinge vorhaben, und „Schweig, frecher Bengel!“, wenn ein junger Mensch zu kritisieren wagt.

\section{Hamburger Morgenpost, 14.1.2015: 8; MEIN WALKMAN, DER WAR WAHNSINN}

Als ich klein war, hab' ich während langer Auto- und Bahnfahrten immer Musik auf meinem Walkman gehört. Lesen fand ich auch super, nur mein Magen leider nicht - und mein Bruder, der immer neben mir saß, dann auch überhaupt nicht. Gestern hatte ich einen siebenjährigen Bengel neben mir in der S-Bahn sitzen. «Papa, ich hab nur 3G», quengelt er und fuchtelt nervös mit dem iPhone herum. Das langsame Internet scheint inn richtig fertigzumachen. Und Papa? Der hört die Hilferufe seines Sohnes gar nicht. Der hat nämlich mehr als $3 \mathrm{G}$ und versinkt im Smartphone. Und ich denke im Stillen an meinen Walkman.

\subsubsection{Flegel}

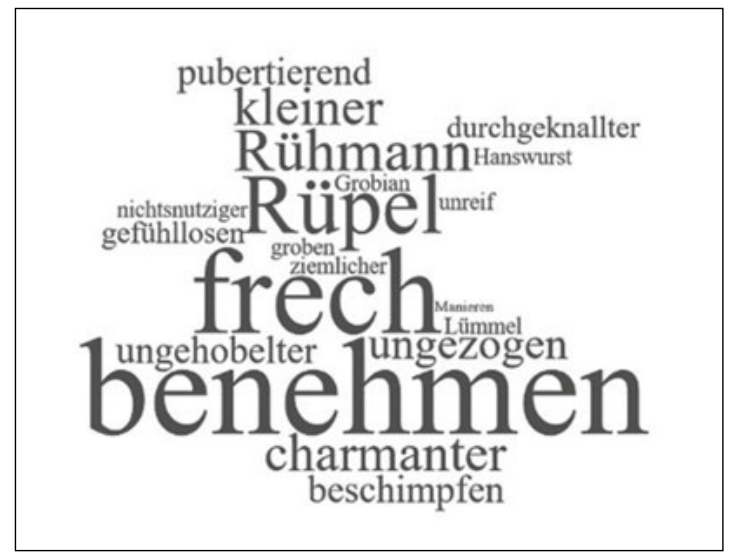

Abb. 7: Flegel Kookkurrenzen 
Der Ausdruck Flegel tritt häufig gemeinsam mit dem Benehmen auf; als wichtigste Attribute erscheinen frech und ungezogen; aufschlussreich sind auch die Verbindungen zu: pubertierend und unreif, die den Alterskontext der Jugendphase anzeigen. Die Kookkurrenzen mit gefühllos, durchgeknallt, nichtsnutzig verweisen auf die Dimensionen der Abweichung von erwartbaren Verhaltensnormen. Der Eigenname Rühmann und der Hinweis auf: charmant stehen im Kontext der Filme: So ein Flegel (1934/44) und: Ein charmanter Flegel (1950) mit Heinz Rühmann und Clark Gable in den Hauptrollen.

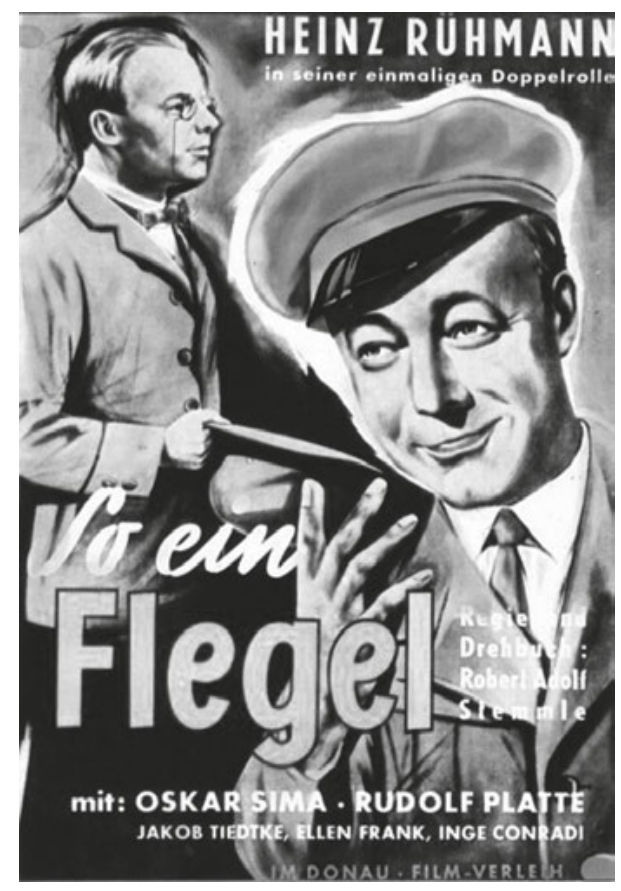

Abb. 8: Filmplakat „So ein Flegel“, Deutschland (1934)

Auch hier seien wieder zwei Pressebeispiele aus der Zeit seit der Jahrtausendwende präsentiert: Die Kontexte verweisen deutlich in die Richtung, die schon in der Wordle-Darstellung ersichtlich wurde. 
Beispiel 2: Flegel:

Neue Zürcher Zeitung, 8.5.2000: 28; Aus der Birne geschüttelt / «König Ubu» in Biel und Solothurn

«Scheisse» ist das erste Wort, das der Flegel namens Ubu spricht, ganz nach der Dramenvorlage von Alfred Jarry. Doch dann ist es weitgehend vorbei mit den Fäkalspässen, und auch sonst hat sich Regisseur Clemens Bechtel in seiner Inszenierung von «König Ubu» für das «ensemble»-Theater der Regionen Biel - Solothurn einige Freiheiten genommen.

Süddeutsche Zeitung, 27.11.2014: 24; Der ewige Staatsbankrott

Lee Buchheit, ein New Yorker Anwalt und Spezialist für die Restrukturierung von Staatsschulden, sagt: „Es ist so, als ob man im Bus seinen Platz für eine gebrechliche ältere Dame räumt, und dann kommt ein halbstarker Flegel daher und fläzt sich hin.“

\subsubsection{Lümmel}

Abb. 9: Lümmel Kookkurrenzen

Bei Lümmel zeigen sich mit den Kookkurrenzen von: Bank, ersten, letzten, Reihe, Pauker deutlich die Einflüsse der Filmtitel: „Die Lümmel von der ersten Bank“. Die Ausdrücke: Heine, Volk verweisen auf den Text von Heines Wintermärchen. Die negativ wertenden Attribute: unverschämt, ungezogen, launisch, arrogant, missraten fügen weitere Bedeutungsdimensionen hinzu, die den unmittelbaren Erziehungskontext transzendieren. 


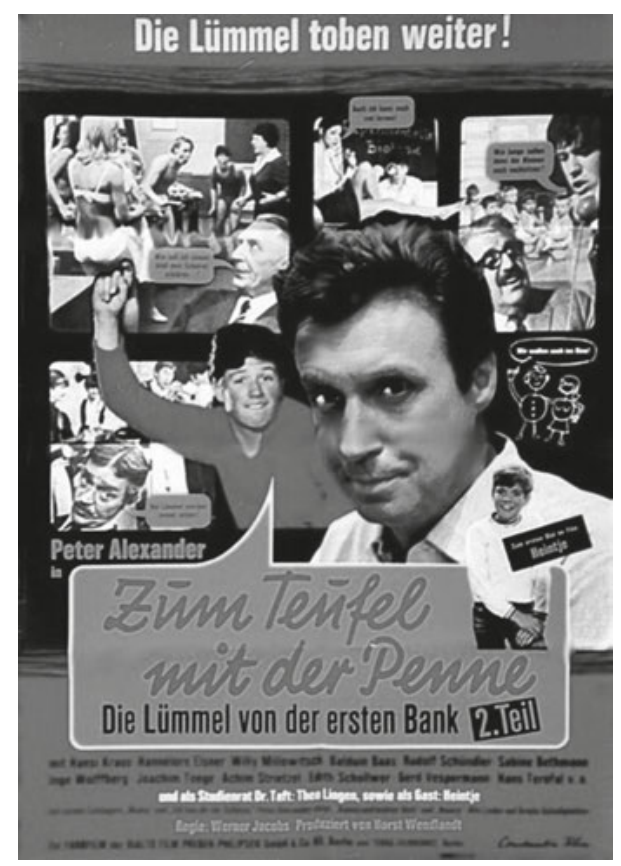

Abb. 10: Filmplakat 2. Teil „Die Lümmel von der ersten Bank“, West-Deutschland (1968)

Die beiden folgenden Pressetexte veranschaulichen, wie schon beim Flegel zu bemerken war, das freche Benehmen der zu Streichen aufgelegten Jugendlichen.

Beispiel 3: Lümmel

\section{Die Zeit, 15.10.1971: 60; Für Filmkunde}

Warum, so muß man fragen, sind Namen wie Jean Renoir, Sergej Eisenstein, Claude Autant-Lara dem Schüler völlig unbekannt? Warum soll ein Schüler nicht die Inhalte der Filmkunst, die Möglichkeiten der Manipulation, die Formenwelt der Filmgeschichte kennenlernen, die er doch tagtäglich am wichtigsten aller Medien verwenden könnte: am Fernsehen. Denn wer Filme von Jean-Luc Godard verstehen gelernt hat, dem droht vom Bildschirmfamilienkriminalserienunsinn keine Gefahr mehr. Doch obwohl fast alle wichtigen Filme im 16-Milimeter-Format vorhanden sind, gammeln die Schulprojektoren still vor sich hin, laufen Schüler massenweise in die „Lümmel von der ersten Bank“, fünfundzwanzigster Teil. 


\section{Braunschweiger Zeitung, 18.10.2007; Zwei Freunde und ihr Zauberwunsch}

Eines Nachts schleichen sie heimlich zum Nachbarhaus. Sie wollen unbedingt Frau

Kleinlich verzaubern. Dauernd schimpft sie mit ihnen, bloß weil sie manchmal ein bisschen laut sind. Anstelle eines Zauberstabs schwingen die beiden frechen Lümmel einen Kochlöffel. „Akrakadabra und Simsalabim!“ Und tatsächlich: Am nächsten Tag hüpft statt Frau Kleinlich ein grüner Frosch in ihrem Garten herum. Oh weiah.

\subsubsection{Rüpel}

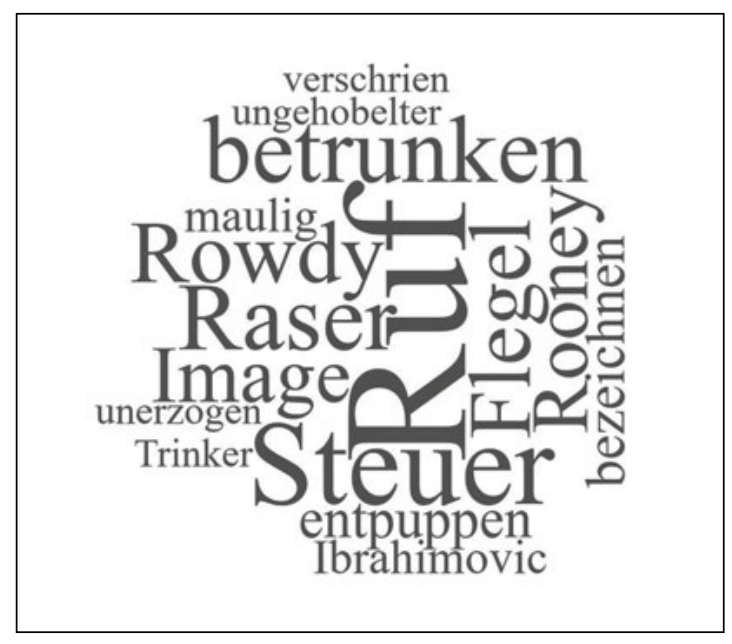

Abb. 11: Rüpel Kookkurrenzen

Im Ruf eines Rowdys oder Flegels zu stehen bzw. ein solches Image zu haben - wie die bekannten Fußballspieler Wayne Rooney und Zlatan Ibrahimovic - scheint eine für die heutige Zeit besonders negative soziale Klassifikation, verbunden mit den Attributen: unerzogen, ungehobelt, maulig. Raser am Steuer entpuppen sich als Rüpel, und auch die Kookkurrenzen: Trinker, betrunken weisen in diese Richtung.

Das Image eines Rowdys wird auch in den beiden beispielhaft ausgewählten Pressetexten thematisiert. 
Beispiel 4: Rüpel

Frankfurter Rundschau, 18.11.1998: 3, Ressort: DIE SEITE 3

Auf die Posse, Jany Le Pen, des Frontführers von jeglicher Politik unberührten Ehefrau, als «Bannerträgerin» an seinem Platz im Europa-Wahlkampf zu erleben, müssen die Franzosen verzichten. Nicht erspart bleibt innen, den Rüpel, der im Wahlkampf eine sozialistische Kandidatin und Bürgermeisterin mit der Faust traktiert, weiter zu sehen.

Die ZEIT, 28.5.2015: 32; Der Spielmacher

Michael Stich entschuldigt sich für einen Schmetterball, der die Sportbürgermeisterin knapp verfehlt. Edwin Weindorfer lässt prüfende Blicke über die Anlage schweifen. Und John McEnroe gibt sich Mühe, das Klischee vom Rüpel auf dem Tennisplatz zu bestätigen. Nach einem missglückten Aufschlag schleudert er seinen Schläger ins Netz. Aber nach dem Spiel wird er freundlich und lobt Stuttgart mit einem breiten Grinsen: «Der Rasen ist hier viel grüner als in Wimbledon!»

\title{
4 Fazit und Ausblick
}

Fassen wir unsere Ergebnisse zusammen, so erhalten wir eine Reihung der sozialen Kategorisierungen „frecher“ Jugendlicher im Hinblick auf die drei folgenden Dimensionen: Alter, Kontext und soziale Wertung:

\begin{abstract}
Alter:
In dieser Dimension nimmt der Ausdruck Bengel die jüngste Position ein, gefolgt vom halbwüchsigen Lümmel, während sich Flegel auf jüngere Männer und Rowdy auch auf Erwachsene beziehen kann.
\end{abstract}

\section{Kontext:}

Die Bezeichnung Bengel tritt oft in familiären Kontexten auf, Lümmel hingegen in schulischen Kontexten. Die Ausdrücke Flegel und Rüpel stehen allgemein für schlechtes Benehmen in der Öffentlichkeit ohne die o.g. Spezifizierungen.

\section{Soziale Wertung:}

Alle vier Kategorien bilden mit ähnlichen Bezeichnungen (siehe Kap. 1) durch die Bedeutungsdimensionen Unerzogenheit, Frechheit, schlechtes Benehmen ein semantisches Feld pejorativer sozialer Klassifikationen. Sie weisen leichte skalare und qualitative Unterschiede auf, die sich bei Bengel und Lümmel eher auf harmlosere Streiche, Frechheiten und Ungezogenheiten, bei Flegel und Rüpel auf schlechtes Benehmen und körperliche Grobheiten beziehen. 
Alle Kategorisierungen sind keine Selbstbezeichnungen, sondern erfolgen aus der Außenperspektive Erwachsener. Wohl auch deshalb finden sich keine Entsprechungen in der aktuellen Jugendsprache. Der lässige Typ der 1960er und 1970er Jahre und der coole Typ von heute mögen zwar auch aus einer Außensicht als unerzogen und frech erscheinen, doch trägt gerade die Unbekümmertheit um gesellschaftliche Konventionen zur positiven Wertung aus der Sicht Jugendlicher bei. Veränderungen in den Erziehungsvorstellungen und differentielle soziale Wertungen zwischen den Generationen haben dazu beigetragen, den empörten Ausruf „Sie Lümmel, Sie!“ mit Patina zu überziehen.

Um abschließend auf den titelgebenden Ausruf zurückzukommen und den lexikalischen, semantischen und soziolinguistischen Bemerkungen noch eine pragmatische Perspektive hinzuzufügen, sei noch der Frage nachgegangen, ob die untersuchten Ausdrücke als „verletzende Worte“ (vgl. Herrmann/Krämer/ Kuch (Hgg.) 2007) und im sprachlichen Kontext eines Ausrufs als Beleidigungen gelten können (vgl. auch Meibauer 2016). Möglich wären in diesem Fall noch „Sie Flegel/Sie Rüpel, Sie!“, nicht aber eine entsprechende Formulierung mit Bengel.

Die „Beleidigung“ ist allerdings keine linguistisch wohldefinierte Kategorie und zudem, wie auch das „Schimpfwort“, stark von kulturellen Differenzierungen und kulturellem Wandel abhängig (vgl. Neuland/Könning/Wessels 2017). Die neuere linguistische Höflichkeitsforschung verwendet dem Brown/LevinsonParadigma (vgl. Brown/Levinson 2007) folgend die Bezeichnungen face threatening bzw. face insulting act, und man könnte zurecht bezweifeln, ob bei einem solchen Ausruf eine gesichtsverletzende Intention angenommen werden kann.

Nimmt man noch die Rolle der möglichen Prosodie hinzu, so ist demgegenüber illokutiv wie auch perlokutiv ein solcher expressiver Ausruf eher als Tadel bzw. Zurechtweisung zu verstehen. Es handelt sich zudem um einen responsiven Sprechakt, in Folge einer als negativ und normverletzend verstandenen Handlungsweise oder verbalen Äußerung. Responsivität und Offensivität schließen sich allerdings nicht aus: Die Verwendung der demonstrativen Personaldeixis in der Distanz anzeigenden pronominalen Sie-Form (Sie $x$, Sie!) trägt zweifellos zu einer offensiv-abwertenden Bedeutung bei, indem der Angesprochene als einer negativ stereotypisierten sozialen Gruppe zugehörig identifiziert bzw. im Sinne Garfinkels (2007) degradiert wird.

Dies gilt allerdings nicht generell, sondern unter bestimmten sozialen und kulturellen Gegebenheiten. Ein Ausruf wie „Sie Lümmel, Sie!“ mag im heutigen intergenerationellen Kontext seine Schärfe verloren haben und eher den Sprecher als antiquitierten Vertreter vergangener Epochen identifizieren. 


\section{Literatur}

Brown, Penelope/Levinson, Stephen (2007): Gesichtsbedrohende Akte. In: Herrmann/Krämer/ Kuch (Hgg.). 59-89.

Busch, Wilhelm (1865): Max und Moritz. München: Braun \& Schneider.

Finkbeiner, Rita/Meibauer, Jörg/Wiese, Heike (Hgg.) (2016): Pejoration. (= Linguistik aktuell 228). Amsterdam/Philadelphia: Benjamins.

Garfinkel, Harold (2007): Bedingungen für den Erfolg von Degradierungszeremonien. In: Herrmann/Krämer/Kuch (Hgg.). 49-59.

Herrmann, Steffen/Krämer, Sybille/Kuch, Hannes (Hgg.) (2007): Verletzende Worte. Die Grammatik sprachlicher Missachtung. Bielefeld: Transcript.

Meibauer, Jörg (2016): Slurring as insulting. In: Finkbeiner, Rita/Meibauer, Jörg/Wiese, Heike (Hgg.): Pejoration. (= Linguistik aktuell 228). Amsterdam/Philadelphia: Benjamins. 145-166.

Neuland, Eva (2017): „Höflichkeit? Respekt!“ Jugendtypische Umgangsformen mit sprachlicher Höflichkeit. In: Ziegler, Arne (Hg.): Jugendsprachen. Aktuelle Perspektiven internationaler Forschung. Berlin: De Gruyter.

Neuland, Eva/Könning, Benjamin/Wessels, Elisa (2017): Zum Umgang mit (Un)Höflichkeit in generationeller Perspektive. In: Ehrhard, Claus/Neuland, Eva (Hgg.): Sprachliche Höflichkeit. Historische, aktuelle und künftige Perspektiven. Tübingen: Narr.

Neuland, Eva (2013): Höflichkeit bei Jugendlichen heute: Widerspruch oder Wandel? In: Grote, Michael et al. (Hgg.): Perspektiven. Das IX. Nordisch-Baltische Germanistentreffen in Oslo/Bergen, 14.-16. Juni 2012. Stockholm: Stockholm University Press. 63-78.

Schegloff, Emanuel (2007): A Tutorial on Membership Categorization. In: Journal of Pragmatics 30. 462-482.

Spreckels, Janet (2006): Britneys, Gangschta und wir. Identitätskonstitution in einer Mädchengruppe. Eine ethnographisch-gesprächsanalytische Untersuchung. (= Variolingua 30). Frankfurt a.M. u.a.: Lang.

\section{Wörterbücher und Korpora}

Deutsches Referenzkorpus (DEREKo) (2015-II): https://cosmas2.ids-mannheim.de (Stand: 27.3.2017).

Duden Online-Wörterbuch (2017): www.duden.de (Stand: 7.2.2017).

Grimm, Jacob/Grimm, Wilhelm (1971): Deutsches Wörterbuch. 16 Bde. in 32 Teilbänden. Leipzig 1854-1961. (Online-Version vom 7.2.2017). Leipzig: Hirzel.

Henne, Helmut/Objartel, Georg (Hgg.) (1825/1984): Bibliothek zur historischen deutschen Studenten- und Schülersprache. 3 Bde. Berlin/New York: De Gruyter.

Kluge, Friedrich (1895/1984): Deutsche Studentensprache. In: Henne/Objartel (Hgg.). 93-236. Küpper, Heinz: (1970): Wörterbuch der deutschen Umgangssprache. Jugenddeutsch. 3 Bde. Hamburg: Claassen.

Küpper, Heinz (1982): Illustriertes Lexikon der deutschen Umgangssprache. Stuttgart: Klett. Seebold, Elmar (Bearb.) (2002): Kluge. Etymologisches Wörterbuch der deutschen Sprache. 24. Aufl. Berlin/New York: De Gruyter. 
Schuchardt (1825/1984): Studentikoses Conversationslexicon. Leipzig. In: Henne/Objartel (Hgg.). 121-190.

Vollmann, Johann (1969/1846): Burschicoses Wörterbuch. Graz: Verlag für Sammler.

Wahrig-Burfeind, Renate (Hg.) (2014): Wahrig Wörterbuch der deutschen Sprache. 2. Aufl. München: Wissenmedia. 